Research

Synthesis Methods

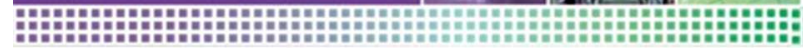

\title{
Evidence Flowers: An Innovative, Visual Method of Presenting 'Best Evidence' Summaries to Health Professional and Lay Audiences
}

\begin{tabular}{|c|c|}
\hline Journal: & Research Synthesis Methods \\
\hline Manuscript ID & RSM-06-2017-0065.R1 \\
\hline Wiley - Manuscript type: & Research Article \\
\hline Date Submitted by the Author: & $\mathrm{n} / \mathrm{a}$ \\
\hline Complete List of Authors: & $\begin{array}{l}\text { Babatunde, Opeyemi; Keele University, Research Institute for Primary Care } \\
\text { \& Health Sciences } \\
\text { Tan, Valerie; Keele University, Research Institute for Primary Care \& Health } \\
\text { Sciences } \\
\text { Jordan, Joanne; Keele University, Research Institute for Primary Care \& } \\
\text { Health Sciences } \\
\text { Dziedzic, Krysia; Keele University, Research Institute for Primary Care \& } \\
\text { Health Sciences } \\
\text { Chew-Graham, Carolyn; Keele University, Research Institute for Primary } \\
\text { Care \& Health Sciences; West Midlands CLAHRC and South Staffs and } \\
\text { Shropshire Foundation Trust, - } \\
\text { Jinks, Clare; Keele University, Research Institute for Primary Care \& Health } \\
\text { Sciences } \\
\text { Protheroe, Joanne; Keele University, Institute of Primary Care and Health } \\
\text { Sciences } \\
\text { van der Windt, Danielle; Keele University, Research Institute for Primary } \\
\text { Care \& Health Sciences }\end{array}$ \\
\hline Manuscript Keywords: & $\begin{array}{l}\text { Evidence based practice, Disemmination, Accessibility, Evidence synthesis, } \\
\text { Clinical guidelines }\end{array}$ \\
\hline Keywords to match reviewers: & $\begin{array}{l}\text { Epidemiology, Public Health, Heath Services Research < Substantive Areas, } \\
\text { Knowledge Exchange < General Methods < Methods Areas, Other < } \\
\text { Substantive Areas }\end{array}$ \\
\hline Abstract: & $\begin{array}{l}\text { Background \& Aims: Barriers to dissemination and engagement with } \\
\text { evidence pose a threat to implementing evidence-based medicine. } \\
\text { Understanding, retention and recall can be enhanced by visual presentation } \\
\text { of information. The aim of this exploratory research was to develop and } \\
\text { evaluate the accessibility and acceptability of visual summaries for } \\
\text { presenting evidence syntheses with multiple outcomes to professional and } \\
\text { lay audiences. } \\
\text { Methods: 'Evidence flowers' were developed as a visual method of } \\
\text { presenting data from four case scenarios: two complex evidence } \\
\text { syntheses, Cochrane reviews and clinical guidelines. Petals of evidence } \\
\text { flowers were coloured according to the GRADE evidence rating system to } \\
\text { display key findings and recommendations from the evidence summaries. }\end{array}$ \\
\hline
\end{tabular}


Page 1 of 24

Research Synthesis Methods

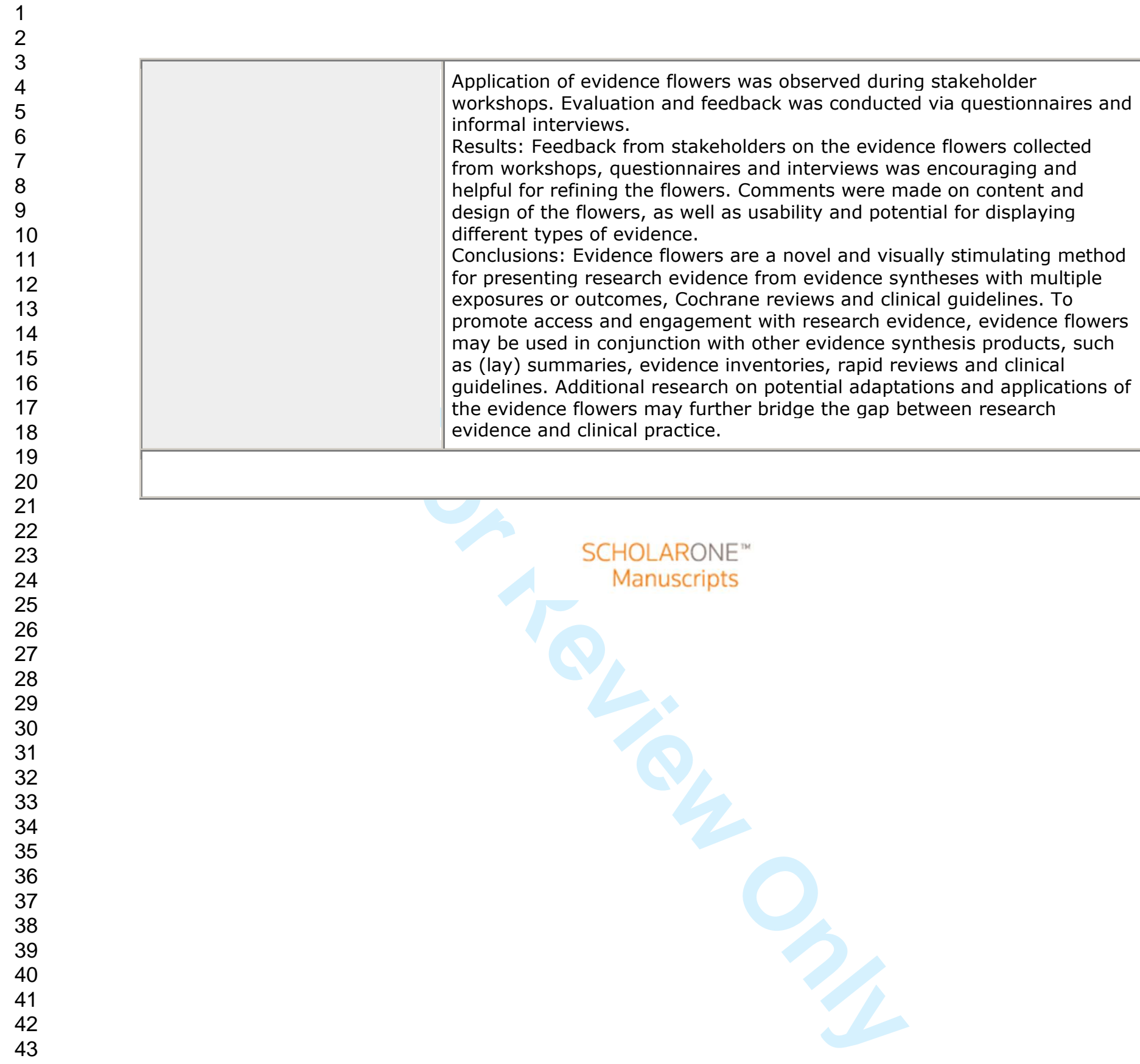

21

22

23

24

25

26

27

28

29

30

31

32

33

34

35

36

37

38

39

40

41

42

43

Application of evidence flowers was observed during stakeholder workshops. Evaluation and feedback was conducted via questionnaires and informal interviews.

Results: Feedback from stakeholders on the evidence flowers collected from workshops, questionnaires and interviews was encouraging and helpful for refining the flowers. Comments were made on content and design of the flowers, as well as usability and potential for displaying different types of evidence.

Conclusions: Evidence flowers are a novel and visually stimulating method for presenting research evidence from evidence syntheses with multiple exposures or outcomes, Cochran reviews and clinical guidelines. To promote access and engagement with research evidence, evidence flowers may be used in conjunction with other evidence synthesis products, such as (lay) summaries, evidence inventories, rapid reviews and clinical guidelines. Additional research on potential adaptations and applications of the evidence flowers may further bridge the gap between research evidence and clinical practice.

44

45

46

47

48

49

50

51

52

53

54

55

56

57

58

59

60 

Visual Method of Presenting 'Best Evidence' Summaries to Health Professional and Lay Audiences

Babatunde OO*, Tan V*, Jordan $\mathrm{JL}^{*}$, Dziedzic $\mathrm{K}^{*}$, Chew-Graham $\mathrm{CA}^{*}$, Jinks C*, Protheroe $\mathrm{J}^{*}$, van der Windt DA*

\author{
Author affiliation and address: \\ *Arthritis Research UK Primary Care Centre \\ Research Institute for Primary Care \& Health Sciences, Keele University \\ \# West Midlands CLAHRC and South Staffs and Shropshire Foundation Trust. \\ OCTOBER 2017
}

Competing interests: None to declare

\author{
Corresponding Author: Dr. Opeyemi O Babatunde \\ Arthritis Research UK Primary Care Centre \\ Research Institute for Primary Care \& Health Sciences, Keele University \\ Staffordshire, ST5 5BG \\ o.babatunde@keele.ac.uk \\ Tel: 01782 733927; Fax: 01782734719 \\ http://www.keele.ac.uk/pchs/
}




\section{Abstract}

Background \& Aims: Barriers to dissemination and engagement with evidence pose a threat to implementing evidence-based medicine. Understanding, retention and recall can be enhanced by visual presentation of information. The aim of this exploratory research was to develop and evaluate the accessibility and acceptability of visual summaries for presenting evidence syntheses with multiple exposures or outcomes to professional and lay audiences.

Methods: 'Evidence flowers' were developed as a visual method of presenting data from four case scenarios: two complex evidence syntheses with multiple outcomes, Cochrane reviews and clinical guidelines. Petals of evidence flowers were coloured according to the GRADE evidence rating system to display key findings and recommendations from the evidence summaries. Application of evidence flowers was observed during stakeholder workshops. Evaluation and feedback was conducted via questionnaires and informal interviews.

Results: Feedback from stakeholders on the evidence flowers collected from workshops, questionnaires and interviews was encouraging and helpful for refining the design of the flowers. Comments were made on the content and design of the flowers, as well as the usability and potential for displaying different types of evidence.

Conclusions: Evidence flowers are a novel and visually stimulating method for presenting research evidence from evidence syntheses with multiple exposures or outcomes, Cochrane reviews and clinical guidelines. To promote access and engagement with research evidence, evidence flowers may be used in conjunction with other evidence synthesis products, such as (lay) summaries, evidence inventories, rapid reviews and clinical guidelines. Additional research on potential adaptations and applications of the evidence flowers may further bridge the gap between research evidence and clinical practice. 


\section{Introduction}

With rapidly increasing research output, barriers to accessibility and engagement with research are an emerging threat to evidence-based medicine. ${ }^{1-3}$ In 2010 , over 75 trials and 11 systematic reviews of trials were published daily, ${ }^{4}$ and publication rates have increased since. With a predicted $50 \%$ growth rate in health literature, ${ }^{5}$ distilling the information overload has become necessary for evidence-based medicine to thrive. Given that systematically synthesised evidence may be regarded as the Holy Grail of evidence-based medicine, ${ }^{6}$ evidence based health information in the form of systematic reviews and clinical guidelines have the potential to improve processes of care and patient outcomes. Evidence-based syntheses form the basis for future research and offer healthcare service providers the information needed to implement effective, evidence-based services.

Systematic reviews represent the highest level of research evidence ${ }^{7}$ and provide clinicians with research summaries to help them stay abreast of current evidence-based recommendations, thus informing best practice. However, managing the continually growing number of systematic reviews and clinical guidelines is not feasible for most health professionals. ${ }^{2}$ In addition, technical language, large volumes of text, and difficulties identifying key review findings, deters engagement and implementation of research evidence into practice. $^{2}$ Words used by doctors and health professionals to describe their information supply include "overwhelming, difficult, daunting, choked, despairing, saturation, exhausted, frustrated, dreadful, and unrealistic"; 8,9 "time consuming, demanding and stressful"., 9,10 It appears that for health professionals, the utility of evidence-based information is inversely proportional to the volume of work required to access it. ${ }^{11}$

Research suggests that the 'human bandwidth' or capacity to take in, comprehend, and efficiently synthesize large amounts of new information can be greatly enhanced by visual presentation of information. ${ }^{12-16}$ Similarly, recall, retention and re-use of visual information is significantly greater than that of textual information. ${ }^{12-17}$ These concepts have proved to be increasingly useful for the communication of health information between health care providers and patients. ${ }^{17}$ However, visual presentation of evidence synthesis appears less explored for health professionals, stakeholders and policy makers. Considering these issues, it was proposed that the development of a succinct, visual format of presenting large bodies of synthesised evidence would facilitate accessibility, acceptability, engagement and usability of the research evidence to wider and more varied stakeholder groups. 
The first evidence flowers were designed to visually present the findings from a large complex evidence synthesis of the effectiveness of interventions for treating the five common musculoskeletal pain conditions within the STarT MSK research programme at Keele University (see below). The aim was to find a way to present information contained in extensive summary tables in a format that was engaging, as well as easily and quickly understood by all the research programme's stakeholders (health professionals, researchers, members of the general public and health providers). The specific objectives were to:

i. develop pictorial graphics (evidence flowers) for presenting a succinct overview of evidence syntheses with multiple exposures (e.g. interventions) or outcomes that also indicated the strength of the evidence;

ii. explore the usability, accessibility and acceptability of the evidence flowers to professional and lay audiences with differing needs, experiences, and preferences;

iii. suggest other applications where evidence flowers might be used to summarise evidence syntheses; and

iv. discuss future research to formally evaluate and test evidence flowers.

Four cases, or scenarios, for which evidence flowers were generated to provide visual summaries of evidence, are described in this paper. The first two cases (research programmes consisting of large, complex evidence syntheses) describe the development of the evidence flowers, Cases 3 and 4 are suggestions for other areas where evidence flowers might be applied to aid knowledge transfer to mixed audiences. The development and application of methods used to collate, disseminate and translate the synthesised evidence-based information into knowledge are reported.

\section{Development of the evidence flowers}

Case study 1: Stratification and Targeted Treatment of Musculoskeletal conditions STarT MSK ${ }^{18}$

STarT MSK is a five-year programme of research that aims to improve patient outcomes by using a stratified approach to the management of common musculoskeletal pain presentations. Treatments that are matched to patient risk subgroups (or stratified) on the basis of prognostic information have been shown to improve patient outcomes and/or reduce health care costs in the primary care management of low back pain. ${ }^{19,20}$ To inform the development of treatment strategies matched to patient risk subgroups for a wider range of musculoskeletal pain presentations, an overview and critical appraisal of current best 
evidence of the available treatment options used in primary care for common musculoskeletal conditions was conducted. ${ }^{21}$ These evidence summaries of treatment effects were then used by stakeholder and expert consensus groups to identify the most promising treatments for the different prognostic subgroups.

\section{Case study 2: Improving care for people with long term conditions - ENHANCE $E^{22,23}$}

The aim of the ENHANCE study was to develop and test the feasibility and acceptability of a complex intervention for tackling the under-recognition and suboptimal management of anxiety and/or depression and osteoarthritis (OA)-related joint pain in patients with long term conditions (LTCs) in primary care. Developing the enhanced consultation and the training for practice nurses for the ENHANCE study required synthesis of the evidence on effective methods for identification and management of anxiety, depression and OA-related joint pain in primary care. Guidelines, systematic reviews and other available evidence concerning the primary care management of the four LTCs (Asthma, Chronic Obstructive Pulmonary Disease (COPD), Cardiovascular Disease/Hypertension, and Type 2 Diabetes) were also reviewed.

\section{Methods}

Development of the evidence flowers was an iterative process that followed the principles of contextual design ${ }^{24}$ and co-design ${ }^{25}$. Evaluation and feedback techniques were used to observe the use of the evidence flowers and collect comments from the diverse groups involved in order to refine the design of the evidence flowers for presenting research evidence.

The large, independent evidence syntheses conducted for STarTMSK and ENHANCE research programmes, comprised of systematic searches of bibliographic databases (including Cochrane database of systematic reviews, Database of abstracts of reviews of effects, Health Technology Assessment database, MEDLINE and EMBASE (using specific search filters to retrieve systematic reviews and clinical guidelines)), and evidence sources (Clinical Knowledge Summaries, Map of Medicine, TRIP Database, reference lists of included systematic reviews and guidelines, research stakeholders and experts in the field) for the most recent, high quality reviews, clinical guidelines and policy documents. The evidence syntheses used pre-appraised evidence, such as clinical guidelines, policy documents, clinical evidence pathways and evidence summaries, wherever possible as the starting point due to 
the broad topic areas being addressed, similar to the 6 s pyramid model described by ${ }^{26}$. The methods and results of these syntheses will be published elsewhere.

Information from evidence syntheses can be complex with multiple aspects to consider. Risk of bias and confounding, strength of recommendations, design of primary sources of data, magnitude of effect, level of precision, directedness, the dose response gradient of evidence, and consistency of results across sources of evidence, may all affect interpretation of the evidence. Grading of Recommendations Assessment, Development and Evaluation (GRADE) takes all these points into consideration by downgrading evidence where these issues arise 27,28 . GRADE criteria is often applied by systematic reviewers and guideline developers to indicate the strength of the body of evidence supporting summary statements or recommendations. Therefore, GRADE seemed a practical system on which to base the evidence flowers.

The strength of evidence in both STarTMSK and ENHANCE syntheses was assessed using a modified GRADE approach. Given the varying study types and different levels of evidence being synthesised, GRADE levels of evidence were modified and applied consistently and in a standardised format across the available sources of evidence. For each treatment option (in STarTMSK) or condition (in ENHANCE), strength of evidence was classified as:

- "Gap in evidence" - Where no published or unpublished evidence has been found

- "Weak evidence" - based solely on expert opinion or consensus in guidelines only or in the absence of systematic review evidence

- "Limited evidence" - in the presence of little evidence from systematic reviews/evidence-based guidelines AND when there were small, inconsistent, or nonsignificant treatment effect sizes from primary studies (trials)

- "Moderate evidence" - in the presence of little evidence from systematic reviews/evidence-based guidelines (as in 2) but showing a medium to large treatment effect in trials OR in the presence of strong evidence from high quality systematic reviews, but with small or inconsistent treatment effect sizes

- "Strong evidence" - in the presence of strong evidence from high quality systematic reviews and evidence-based clinical guidelines AND medium or large effect sizes from trials.

Use of a flower design enabled presentation of a number of different treatment options or different outcomes to be represented (depending on the type of evidence presented) as petals, with the colour of the petal representing the GRADE rating of the evidence supporting each 
option. (Fig. 1). The results of evidence syntheses were summarised and evidence flowers produced. The synthesised evidence was subdivided such that each flower contained information relative to a specific health condition or area of interest (for example, see the summary of evidence for treatments of cervical radiculopathy in STarT MSK; and for casefinding and assessment of osteoarthritis in multimorbidity for ENHANCE, Fig. 2). The petals represented the treatment or management options available and the colour indicated the GRADE rating of the evidence. The resulting evidence flowers formed a simple representation of the strength of current best evidence and a summary of the evidence syntheses (Fig. 2).

The evidence flowers were used alongside traditional evidence summary tables, which were also available for consultation when more detailed information was required. Both the evidence flowers and the summary tables were presented to stakeholder groups (consisting of policy decision makers, commissioners, health service managers, clinicians, and patient representatives) in a series of workshops that aimed to facilitate dissemination of current best evidence and inform the development of the next stage in each research programme.

For the STarT MSK programme, the stakeholder workshops focused on identifying effective treatment options (based on findings from the evidence syntheses), which would be most suitable for patient risk subgroups (i.e. patients at low, medium, or high risk of persistent, disabling pain). After the workshop, evidence flowers were revised according to consensus opinion, developing and adapting them to facilitate dissemination of evidence to the wide variety of end users. The revised flowers were then used in a series of expert consensus group meetings to determine matched treatment options for the STarT MSK stratified care trial.

Accessibility, acceptability and engagement were explored via structured questionnaires (Appendix 1). The questionnaires were adapted from existing studies ${ }^{29,30}$ and required responses from participants regarding experience of interpreting research evidence and evidence-based medicine skills, recognition and understanding of key messages presented in the evidence flowers, ease of access to and perceived value of the evidence flowers, and preferred summary formats. The questionnaires were reviewed (pilot-tested) by local researchers and clinicians.

A similar process was observed during the initial phases of the ENHANCE trial, during which the evidence flowers were used to stimulate discussion at three separate stakeholder workshops, ultimately facilitating development of the new complex intervention. Evidence 
flowers were not formally assessed using questionnaires for the ENHANCE stakeholder workshops. However, feedback from stakeholders not only informed modifications to the evidence flowers, but was also used to refine the questionnaires used in the STarTMSK programme to gather more specific information during subsequent workshops.

\section{Results of evidence flowers evaluation and feedback}

In the ENHANCE study, feedback was positive (Table 1), including critical comments regarding concept, design, content and usability of the evidence flowers for presenting different types of evidence, or to different audiences. Informal feedback was gathered from stakeholders and experts who indicated interest in being contacted for further discussion a few weeks after the workshop.

Discussions focused on collecting information about participants' experiences of using the evidence flowers for accessing synthesised evidence, their own use of research evidence and views on potential use of evidence flowers in research, policy and clinical practice. The concise content presented through the evidence flowers was considered to be a strength. There were discussions about standardising the amount of text on all petals representing different treatment options. Regarding strength of evidence, the GRADE rating system was generally well accepted but there were conflicting comments about indicating the direction (i.e., positive or negative) of treatments effects on evidence flowers, and specification of efficacy versus effectiveness information on the petals. A wide range of potential applications and usefulness in research, clinical practice and patient education were highlighted in discussions, this needs to be explored and tested in future studies.

To evaluate the flowers, participants were asked to complete questionnaires at the end of the STarT MSK stakeholder workshop. Twenty-one people (eleven clinicians, five academic researchers, three epidemiologists, and two health service/trial managers) responded to the questionnaire out of 22 eligible attendees. In total, 27 people attended the workshop, but five were researchers who were either involved with facilitating the workshop or directly involved in the evidence synthesis/development of evidence flowers and therefore not eligible). Out of 21 responders, 18 found it easy to understand visual presentation of evidence in form of evidence flowers, while three out of 21 were not certain evidence flowers helped them to carry out set tasks for the stakeholder workshop. Nearly half, nine out of 21 thought changes could be made to evidence flowers to further enhance its usability. Details of responses to questionnaire items for the STarT MSK stakeholder group are presented in Table 1. 
The development and concept of the evidence flowers in these two cases as visual representation of complex evidence syntheses was presented at scientific conferences. This provided national and international opportunities to stimulate further debate and inform dialogue with a wider audience around how to translate synthesised evidence into clinical practice and policy. ${ }^{31-33}$

\section{Potential Applications}

\section{Case study 3: Cochrane reviews}

Whilst widely recognized as a gold standard of evidence-based health care resources, Cochrane reviews are not without accessibility problems for health care professionals. ${ }^{29}$ Despite potential solutions, such as characteristics of studies and Summary of Findings tables, to facilitate the uptake of information from Cochrane reviews, statistical information and large volumes of text results remain difficult to read and interpret quickly potentially limiting their use for health professionals in clinical practice.

Alternative methods of visually presenting the Cochrane evidence may improve accessibility. It was hypothesised that when used in conjunction with Summary of findings tables, evidence flowers may make evidence more accessible to a variety of audiences. For five Cochrane reviews containing summary of findings tables (purposively selected to represent a range of topic areas or specialties), evidence flowers were created. ${ }^{34}$ The petals of the evidence flowers represented evidence of the effectiveness of treatment for different outcome measures, and were coloured according to the GRADE evidence rating system given by the Cochrane review authors in the published Summary of Findings tables. The standardised narrative summary on the petal was kept brief and written in plain language (Fig. $\underline{3}$ ). Effect sizes were indicated with a three-star grading system classifying effect sizes as small $(d=0.2)$, medium $(d=0.5)$, or large $(d \geq 0.8)$ (Carson).

\section{Case study 4: Evidence-based clinical guidelines}

Despite their potential to improve health care outcomes, clinical guidelines have been plagued with wide variations in implementation in clinical practice across health care professions and disease spectrums. ${ }^{35}$ Major barriers to guideline implementation include large volumes of text to read, time required to access documents, unclear formats of the guidelines and lack of motivation. ${ }^{1,10,35}$ Given that their impact is reliant on successful implementation, 
the question remains as to how clinicians stay abreast of core recommendations and remain motivated to implement these in their practice. The feasibility of expressing core guideline recommendations in form of evidence flowers for improving stakeholder accessibility and engagement was explored.

Two National Institute for Health and Care Excellence (NICE) guidelines, Depression in Adults $^{36}$ and Osteoarthritis, ${ }^{37}$ were selected and evidence flowers representing core $\underline{\text { recommendations of the guidelines were created using an iterative process. This involved }}$ extracting relevant information into summary tables (Appendix 2), highlighting multiple clinical outcomes considered in the guideline, and guideline recommendations for management. Information to be included in the evidence flowers was subsequently generated using data from the evidence summary tables. As with previous case studies, the strength of the research evidence supporting each recommendation was indicated by using different coloured petals in the evidence flowers according to the grading (based on GRADE) allocated by the guideline development group. The narrative summary of the information on the petal was kept brief and written in plain language (Fig $\underline{4})$.

Two members of the NICE working groups, who had been involved with the development of these guidelines, gave informal feedback to ensure agreement of the evidence flowers with the evidence published in the guidelines. Accessibility and acceptability of the evidence flowers for use in this context were also considered.

Feedback and discussions centred mostly on reconciliation between the grade of evidence and the guideline working group recommendations to offer or not to offer particular treatment. For instance, despite the low quality of evidence in support of the effectiveness of patient education and advice for the management of osteoarthritis (Fig. 4), the recommendation of the guidelines group (based on consensus, clinical expertise and ethics in practice) was to offer patient advice and education as a core recommendation. Here, visual presentation with evidence flower suggests that lack of research evidence may not always be interpreted as having limited value in practice. It highlights the evidence flower's potential to offer accessible summaries of complex evidence-based health information provided in clinical guidelines.

\section{Discussion}


In an era where the call for better standards of care through the use of evidence-based healthcare is unprecedented, and health care professionals are expected to keep up to date with an ever increasing amount of health care evidence, there is an urgent demand for evidence-based syntheses that are accessible to a wide variety of audiences. The four evidence flower case descriptions presented in this paper are examples of how simple visual summaries of synthesised evidence may be used to display complex information.

In the review by Dawes and Sampson, ${ }^{38}$ information seeking is more likely to occur and be successful if access is convenient, information is reliable, of high quality, applicable and can be put into practice quickly. Engagement with research evidence must be preceded by accessibility to the evidence. The evidence flowers were found in these cases to be a suitable method for presenting the results of evidence syntheses to various stakeholders with varying levels of research experience as well as in forums with conference audiences.

Engagement and accessibility barriers related to the speed of use, recall and retention of information contained in systematic review and clinical guidelines were considered, at least partly, overcome with the use of evidence flowers. For instance, during the workshops, the stakeholders seemed to quickly perceive the overall effect and direction of evidence supporting each treatment and were able to refer to and engage with more detailed information in the supporting evidence tables and reports. Much of the synthesised evidence presented contained no meta-analysis and it was not possible to extract statistical summary estimates of treatment effects to include such information in the evidence flowers. Given the $\underline{\text { main aim of the flowers, which was to offer an overview of the evidence that is accessible to }}$ lay members of the public and healthcare professionals, the absence of quantitative data in the flowers was not considered a key issue. When discussing the evidence as presented in the flowers, our groups could access more detailed evidence tables that included summary estimates where available. Further testing with end-users should formally assess whether conclusions drawn from the evidence flowers alone is similar to conclusions when using evidence tables, and provides a correct and unbiased interpretation of the evidence. Evidence flowers were perceived by most participants in the stakeholder workshops to have helped in stimulating discussions around the body of evidence. Visual presentation of information, such as the evidence flower, may be useful as discussion starters in clinical practice or research settings, and health implementation science forums.

Criticisms about the appearance of evidence flowers related to size and colour palette. Discussants weighed the pros and cons of varying the size of petals in relation to the strength 
of evidence. Further research and development will explore the impact of varying the size of the impact of varying the size of the petals in proportion to strength of evidence or the magnitude of effects. Based on the cases presented in this paper, 5-6 petals appear to be the optimal number of petals feasible for printed versions of the evidence flowers. Pragmatically, future adaptations of evidence flowers may need to be more suitable for online /web applications as this would also allow for adequate adjustment for size and shape of petals.

Research has shown that colours have effect on attention, memory and recall. ${ }^{39}$ The current colour scheme was arbitrarily chosen to indicate contrast between each of the levels of evidence. Feedback has included issues such as "colours are too feminine", and the intuitive association of lighter colours with low quality evidence versus darker colours with high quality evidence by users. In order to enhance accessibility and acceptability, future development and application of the evidence flowers will benefit from a careful consideration of the colour palette used to represent the different grades of evidence and the effect this has on their interpretation and impact in different contexts and audiences.

The initial design for the evidence flowers was manually created using standard software (MS Office) which is not optimal for efficiently generating the flowers in terms of fitting content, and increasing number or size of petals. Furthermore, given that evidence-based health information needs to be updated regularly, consideration needs to be given to ensuring

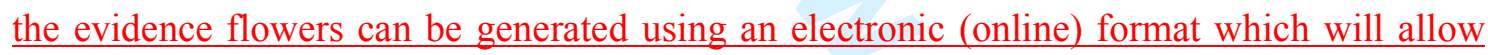
easy formatting and updating of the evidence flowers, and offer links to more detailed evidence behind each recommendation if required.

Evidence flowers were initially developed within the context of two specific research programmes when the need emerged to present a large, complex body of evidence in a way that was suitable for a wide range of stakeholders. Therefore, evaluation of its potential to enhance accessibility, acceptability and engagement with synthesised research evidence is limited at present to the four case studies reported here. Weaknesses of this exploratory research relates mainly to the small number of participants taking part in workshops and completing questionnaires, as well as the lack of rigorous evaluation methods. However, early results for the use of evidence flowers are promising, and the concept has been generally well received by professionals and lay health audiences in national and international forums where it has been presented. Well-designed research studies evaluating the application of visual presentation of synthesised evidence is an important next step. 
The use of visual information has helped to advance other areas of learning and communication such as marketing, engineering and patient health information systems. ${ }^{13,17}$ Our perception that engagement with research evidence may be enhanced by the use of evidence flowers is supported by previous research showing that a high proportion of people are visual learners. ${ }^{12,15}$ Many people make sense of a visual scene almost a million times faster than text, recognise as well as retain more visual details than text, and it is thought that this may consequently increase the likelihood of recollecting and re-using information.$^{12,15}$ However, the long or short term recollection, interpretation and re-use of evidence presented in the evidence flowers has yet to be formally tested.

The evidence flowers have not yet been explored for presenting evidence summaries to patients and the general public outside the research or conference environment. However, synthesised evidence is increasingly presented directly to the general public in order to increase health literacy and enable them to make shared, evidence-informed health decisions. Further work on the evidence flowers with patients and the public may provide insight into if and how evidence flowers can be used to present the information needed to facilitate shared decision making with their health care providers. Research in the future will also need to include an examination of the reliability and validity of evidence flowers for presenting and interpreting evidence, as well as a more rigorous evaluation of the impact of evidence flowers when used to enhance accessibility uptake of evidence in clinical practice.

\section{Conclusions}

Evidence flowers are a novel and visually stimulating method for presenting research evidence. To promote access to and engagement with research evidence, evidence flowers may be used in conjunction with complex evidence syntheses with multiple exposures or outcomes, Cochrane reviews and clinical guidelines. Further research on potential adaptations and applications to a variety of audiences is planned in order to more thoroughly examine the potential usefulness and impact of using the flowers.

\section{Highlights}

What is already known:

- Technical language, large volumes of text, and difficulties identifying key review findings, deters engagement and implementation of research evidence into practice. 
- With rapidly increasing research output, distilling the information overload has become necessary for evidence-based medicine to thrive.

\section{What is new:}

- To promote access and engagement with research evidence, Evidence flowers has been developed as a visual method of presenting research evidence.

- Evidence flowers has been used to present research evidence from complex evidence syntheses with multiple outcomes, Cochrane reviews and clinical guidelines.

\section{Potential impact for $\mathrm{RSM}$ readers:}

- The use of evidence flower design with petals displaying health information and colours representing the strength of evidence enhances accessibility to succinct research evidence that is also visually appealing for lay and professional audience.

- Future research on potential adaptations and applications of the evidence flowers may further bridge the gap between research evidence and clinical practice. 


\section{Acknowledgements}

- We would like to acknowledge the contributions of our stakeholders.

- Patient and public involvement (PPI): The Arthritis Research UK Primary Care Centre at Keele is committed to taking an explicit and systematic approach to involving patients and the public in research. Members of a Research User Group worked in collaboration with researchers on a wide range of tasks including: development, design and delivery of the studies featured in the manuscript, and stakeholder groups reviewing evidence flowers.

\section{Funding}

- This manuscript presents independent research funded by a Centre of Excellence grant from the Arthritis Research UK (grant number: 20202) and the National Institute for Health Research (NIHR) under its Programme Grants for Applied Research scheme (grant number: RP-PG-1211-20010). CJ, KD, CCG, VT are part-funded by the NIHR Collaborations for Leadership in Applied Health Research and Care West Midlands, and The views expressed in this publication are those of the author(s) and not necessarily those of the NHS, the National Institute for Health Research or the Department of Health or the Arthritis Research UK.

- KD is part-funded by a Knowledge Mobilisation Research Fellowship (KMRF-2014-03002) from the NIHR. 
REFERENCES

1. Cabana MD, Rand CS, Powe NR, et al. Why don't physicians follow clinical practice guidelines? A framework for improvement. JAMA. 1999;282(15):1458-1465.

2. Greenhalgh T, Howick J, Maskrey N, Evidence Based Medicine Renaissance G. Evidence based medicine: a movement in crisis? BMJ. 2014;348:g3725.

3. Straus SE, Tetroe J, Graham ID, eds. Knowledge translation in health care: moving from evidence to practice. 2nd ed: John Wiley \& Sons; 2013.

4. Bastian H, Glasziou P, Chalmers I. Seventy-Five Trials and Eleven Systematic Reviews a Day: How Will We Ever Keep Up? PLoS Med. 2010;7(9): e1000326.

5. Hoffmann T, Erueti C, Thorning S, Glasziou P. The scatter of research: cross sectional comparison of randomised trials and systematic reviews across specialties. BMJ. 2012;344:e3223.

6. Sackett DL, Rosenberg WM, Gray JA, Haynes RB, Richardson WS. Evidence based medicine: what it is and what it isn't. BMJ. 1996;312(7023):71-72.

7. OCEBM Levels of Evidence Working Group. The Oxford Levels of Evidence 2. 2011; http://www.cebm.net/index.aspx?o=5653. Accessed February 2, 2017.

8. Smith R. What clinical information do doctors need? Brit Med $J$. 1996;313(7064):1062-1068.

9. Smith R. Strategies for coping with information overload. BMJ. 2010;341:c7126.

10. Lugtenberg M, Zegers-van Schaick JM, Westert GP, Burgers JS. Why don't physicians adhere to guideline recommendations in practice? An analysis of barriers among Dutch general practitioners. Implement Sci. 2009;4:54.

11. Shaughnessy AF, Slawson DC, Bennett JH. Becoming an information master: a guidebook to the medical information jungle. J Fam Pract. 1994;39(5):489-499.

12. Brady TF, Konkle T, Alvarez GA. A review of visual memory capacity: Beyond individual items and toward structured representations. J Vis. 2011;11(5):4.

13. Carney RN, Levin JR. Pictorial illustrations still improve students' learning from text. Educ Psychol Rev. 2002;14(1):5-26.

14. Chandler P, Sweller J. Cognitive Load Theory and the Format of Instruction. Cognition Instruct. 1991;8(4):293-332.

15. Endress AD, Potter MC. Something from (almost) nothing: buildup of object memory from forgettable single fixations. Atten Percept Psycho. 2014;76(8):2413-2423.

16. Horn RE. Visual language and converging technologies in the next 10-15 years (and beyond). In: Roco MC, Bainbridge WS, eds. Converging Technologies for Improving Human Performance Arlington, VA: National Science Foundation \& Springer; 2002:141-149.

17. Houts PS, Doak CC, Doak LG, Loscalzo MJ. The role of pictures in improving health communication: a review of research on attention, comprehension, recall, and adherence. Patient Educ Couns. 2006;61(2):173-190.

18. Research Institute for Primary Care \& Health Sciences. Musculoskeletal Pain and Stratified 
https://www.keele.ac.uk/pchs/research/musculoskeletalpainandstratifiedcare/.

Accessed October 31, 2017.

19. Hay EM, Dunn KM, Hill JC, et al. A randomised clinical trial of subgrouping and targeted treatment for low back pain compared with best current care. The STarT Back Trial Study Protocol. BMC Musculoskelet Disord. 2008;9:58.

20. Hill JC, Fritz JM. Psychosocial influences on low back pain, disability, and response to treatment. Phys Ther. 2011;91(5):712-721.

21. Babatunde OO, Jordan JL, van der Windt D, Foster NE, Protheroe J. Effective Treatment Options for Musculoskeletal Pain Conditions: A Rapid Meta-Synthesis of Current Best Evidence in Primary Care. Rheumatology (Oxford). 2016;55(Suppl 1):i80-i80.

22. Healey EL, Jinks C, Tan VA, et al. Improving the care of people with long-term conditions in primary care: protocol for the ENHANCE pilot trial. $J$ Comorb. 2015;5(1):135-149.

23. Jinks C, Morden A, Chew-Graham C, et al. Case-Finding for Joint Pain and Anxiety and Depression: The Enhance Study. Ann Rheum Dis. 2015;74(Suppl 2):1182-1182.

24. Holtzblatt K, Beyer H. Contextual Design: Design for Life. Elsevier Science; 2016.

25. Donetto S, Pierri P, Tsianakas V, Robert G. Experience-based Co-design and Healthcare Improvement: Realizing Participatory Design in the Public Sector. Des $J$. 2015;18(2):227-248.

26. Dicenso A, Bayley L, Haynes RB. Accessing pre-appraised evidence: fine-tuning the 5S model into a 6S model. Evid Based Nurs. 2009;12(4):99-101.

27. Guyatt GH, Oxman AD, Kunz R, et al. What is "quality of evidence" and why is it important to clinicians? BMJ. 2008;336(7651):995-998.

28. Guyatt GH, Oxman AD, Vist GE, et al. GRADE: an emerging consensus on rating quality of evidence and strength of recommendations. BMJ. 2008;336(7650):924-926.

29. Rosenbaum SE, Glenton C, Cracknell J. User experiences of evidence-based online resources for health professionals: user testing of The Cochrane Library. BMC Med Inform Decis Mak. 2008;8:34.

30. Rosenbaum SE, Glenton C, Nylund HK, Oxman AD. User testing and stakeholder feedback contributed to the development of understandable and useful Summary of Findings tables for Cochrane reviews. J Clin Epidemiol. 2010;63(6):607-619.

31. Babatunde OO, Jordan JL, van der Windt D, et al. Evidence Flowers: Improving accessibility and engagement with evidence based guidance. Evidence Live; 2016; Oxford, UK.

32. Chew-Graham CA, Jinks C, Tan V, et al. An Innovative, Visual Method of Presenting Summarised 'Best Evidence' To Health Professional and Lay Audiences. World Association of Social Psychiatry (WASP) Jubilee Congress 2014; London, UK.

33. Tan V, Jordan JL, Chew-Graham CA, Jinks C, Babatunde OO, Foster NE. Synthesizing best evidence in 'evidence flowers'. Royal College of General Practitioners (RCGP) Annual Primary Care Conference; 2014; Liverpool, UK. 
34. Jordan JL, Babatunde OO, van der Windt D. Bunches of evidence: displaying 'Summary of findings' tables visually as 'evidence flowers'. 23rd Cochrane Colloquium; 2015; Vienna, Austria.

35. Pronovost PJ. Enhancing physicians' use of clinical guidelines. JAMA. 2013;310(23):2501-2502.

36. National Institute for Health and Care Excellence. Depression in adults: recognition and management. Clinical Guideline [CG90]. London, UK: NICE;2009.

37. National Institute for Health and Care Excellence. Osteoarthritis: care \& mangement. Clinical Guideline [CG177]. London, UK: NICE;2014

38. Dawes M, Sampson U. Knowledge management in clinical practice: a systematic review of information seeking behavior in physicians. Int $J$ Med Inform. 2003;71(1):9-15.

39. Huchendorf L. The effects of color on memory. Journal of Undergraduate Research X. 2007:1-4. 
Table 1. Response to questionnaire \& unedited comments from STarT MSK \& ENHANCE Stakeholder Workshops

\begin{tabular}{|c|c|}
\hline Questions & Response \\
\hline Responders & $\begin{array}{l}21 \text { responders: clinicians (11), researchers (5), Epidemiologists (3), health } \\
\text { service/trial managers (2) }\end{array}$ \\
\hline $\begin{array}{l}\text { 'Evidence flowers' easy to understand? } \\
1=\text { easy; } 2=\text { neutral; } 3=\text { Difficult }\end{array}$ & $18=$ easy; $2=$ neutral; $1=$ Difficult \\
\hline $\begin{array}{l}\text { 'Evidence flowers' help to match the } \\
\text { treatments? } 1=\text { Yes; } 2=\text { No; } 3=\text { Unsure }\end{array}$ & $17=$ Yes; $2=$ No; $3=$ Unsure \\
\hline $\begin{array}{l}\text { Any changes to the appearance of the } \\
\text { flowers? } 1=\text { Yes; } 2=\text { No; } 3=\text { Unsure }\end{array}$ & $9=$ Yes $; 11=$ No; $1=$ Unsure \\
\hline Comments if Yes & $\begin{array}{l}\text { - incorporate visual representation of efficacy } \\
\text { - alter size of petals not just the colour to show evidence level/quality } \\
\text { - colour coding hard to follow } \\
\text { - more summaries of colours in quality and colours for amount of evidence } \\
\text { - make size to show how effective + the colour to represent evidence } \\
\text { - maybe find a way of distinguishing positive and negative evidence more clearly } \\
\text { make all the petals identical }\end{array}$ \\
\hline Changes to the grading system used? & $3=$ Yes; $16=$ No; $2=$ Unsure \\
\hline Comments if Yes & $\begin{array}{l}\text { - Difference between evidence...+efficacy } \\
\text { - Differentiate strength of evidence from quality of evidence } \\
\text { size of petals relate to amount of evidence }+ \text { colour quality }\end{array}$ \\
\hline Changes to the content of the flowers? & $3=$ Yes $; 16=$ No; $2=$ Unsure \\
\hline Comments if Yes & $\begin{array}{l}\text { - useful to be backed up by more detail/explanation of terms on separate sheet as } \\
\text { was the case today } \\
\text { - get rid of extra content } \\
\text { Better format rather than coloured petals? }\end{array}$ \\
\hline $\begin{array}{l}\text { Audience for Evidence flowers? } \\
\text { 1=Patients; } 2=\text { Service providers; } 3= \\
\text { Commissioners; } 4=\text { Healthcare } \\
\text { managers; } 5=\text { Non-academic clinicians; } \\
6=\text { Academic clinicians; } 7= \\
\text { Researchers/Academics }\end{array}$ & $\begin{array}{l}\text { 15-patients, } 17 \text {-service providers, 16-commissioners, } 18 \text {-health care managers, } \\
\text { 19-non-academic clinicians, } 18 \text {-academic clinicians, } 15 \text {-researchers/academics }\end{array}$ \\
\hline
\end{tabular}

\section{Further comments}

\begin{tabular}{|c|c|}
\hline $\begin{array}{l}\text { Comments on Useability \& } \\
\text { Acceptability of Evidence }\end{array}$ & 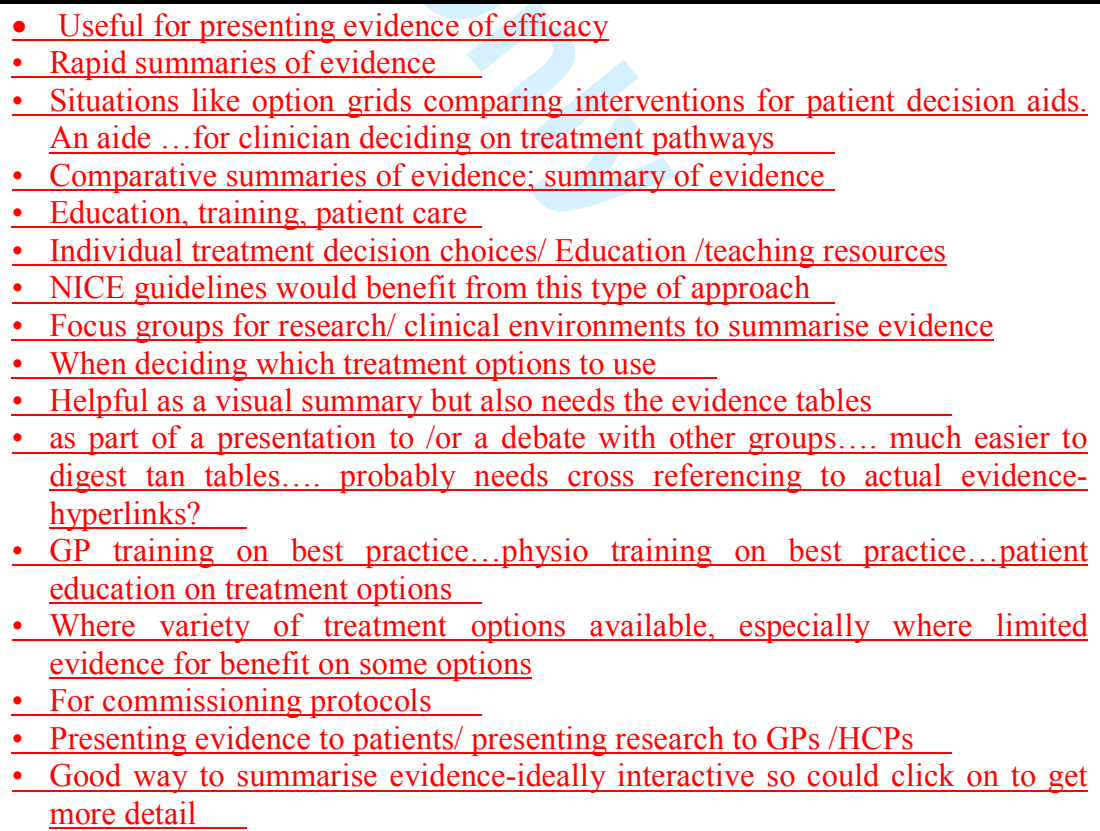 \\
\hline
\end{tabular}




\section{Page 21 of 24}

Research Synthesis Methods

Evidence Flower Manuscript

1

2

3

4

5

6

7

8

9

10

11

12

13

14
15

15

16

17

18

19

20

21

22

23

24

25

26

27

28

29

30

31

32

33

34

35

36

37

38

39

40

41

42

43

44

45

46

47

48

49

50

51

52

53

54

55

56

57

58

59

60

\begin{tabular}{|c|c|}
\hline & $\begin{array}{l}\text { - Useful summary } \\
\text { We have been thinking of a way to our disseminate service audits, this could } \\
\text { probably help } \\
\text { Unclear who the flowers are aimed at - layperson, clinician etc.? }\end{array}$ \\
\hline Copments on Accessibility & $\begin{array}{l}\text { - Good visual representation - able to take evidence in at a glance } \\
\text { - Use as part of presentation to Clinicians /or a debate with other groups....much } \\
\text { easier to digest reports and tables }\end{array}$ \\
\hline $\begin{array}{l}\text { Colmments on Engagement with } \\
\text { evidence }\end{array}$ & $\begin{array}{l}\text { - Could be used in comparative summaries of evidence in training, patient care } \\
\text { - good way to summarise evidence-ideally interactive so could click on to get } \\
\text { more detail } \\
\text {-May be useful for commissioners"; } \\
\text { lexplanation of terms }\end{array}$ \\
\hline
\end{tabular}


Fig 1: Generic evidence flower, GRADE and interpretation of scheme

Fig 1: Generic evidence flower, GRADE and interpretation of scheme

$998 \times 700 \mathrm{~mm}(96 \times 96 \mathrm{DPI})$

Gap in evidence.

Very low quality evidence: We are very uncertain about the estimate.

Low quality evidence: Further research is very likely to have an important impact on our confidence in the estimate of effect and is likely to change the estimate.

Moderate quality evidence: Further research is likely to have an important impact on our confidence in the estimate of effect and may change the estimate.

High quality evidence: Further research is very unlikely to change our confidence in the estimate of effect. 


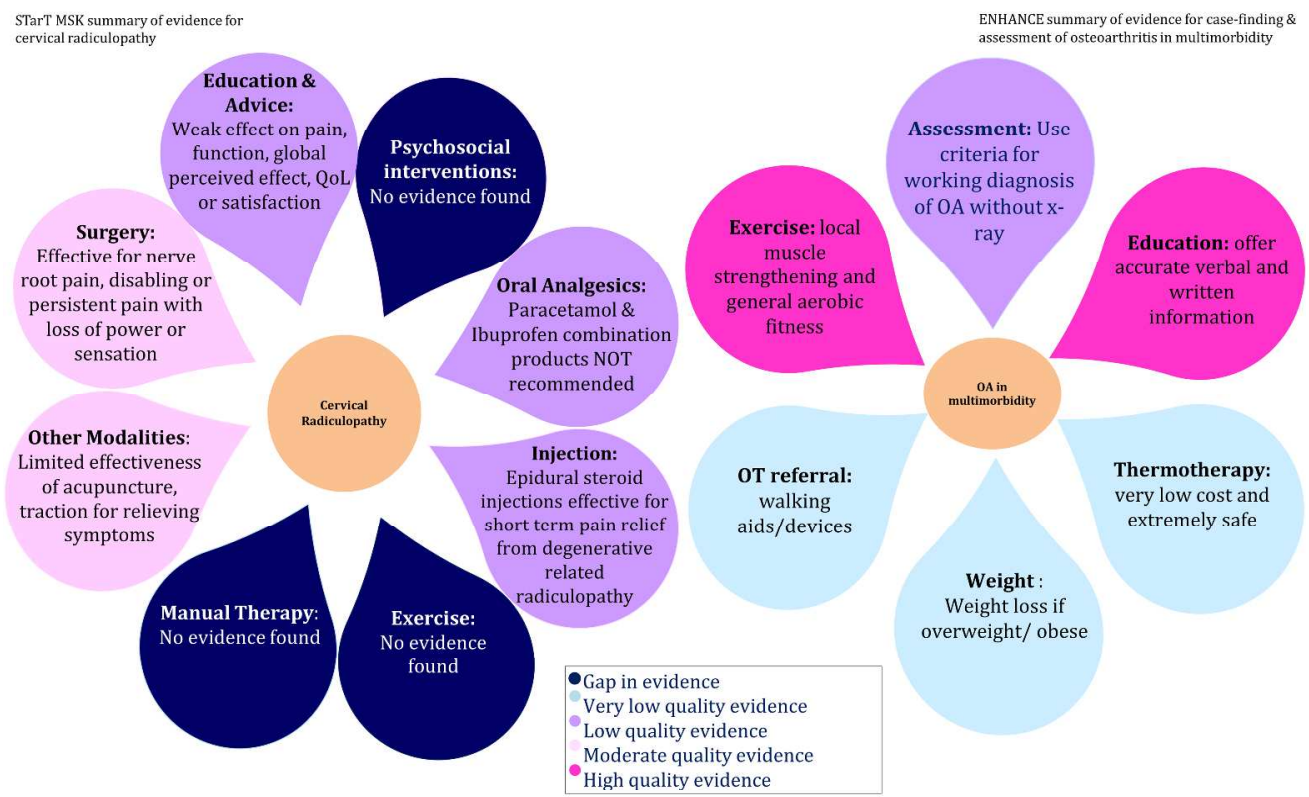

Fig 2: Evidence flower example: STarT MSK summary of evidence for cervical radiculopathy; \&

ENHANCE summary of evidence for case-finding and assessment of osteoarthritis in multimorbidity

Fig 2: Evidence flower example: STarT MSK summary of evidence for cervical radiculopathy; \& ENHANCE summary of evidence for case-finding and assessment of osteoarthritis in multimorbidity 


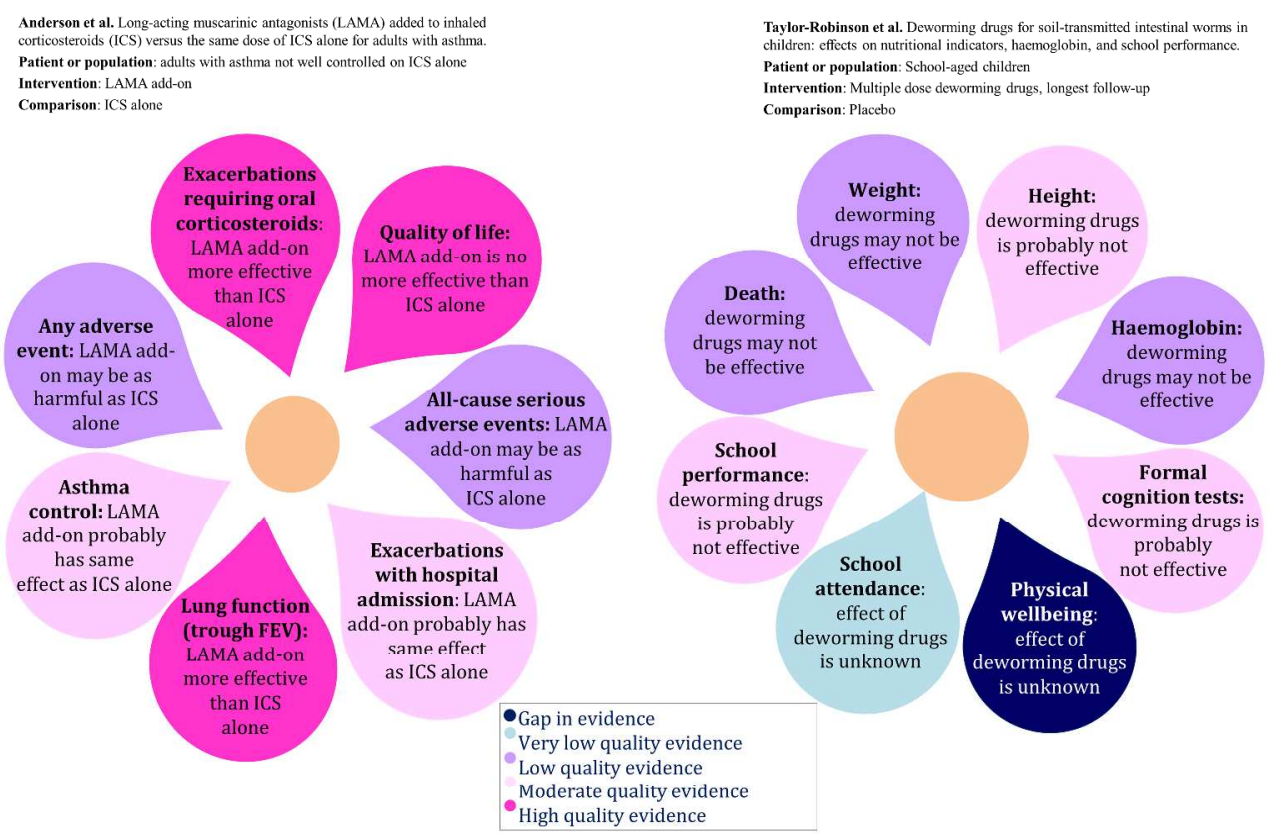

Figure 3. Evidence flowers presenting Summary of Findings tables in two Cochrane reviews -Taylor et al. 2014; Anderson et al. 2015. (23 $3^{\text {rd }}$ Cochrane Colloquium Jordan et al 2015)

Figure 3. Evidence flowers presenting Summary of Findings tables in two Cochrane reviews -Taylor et al. 2014; Anderson et al. 2015.

(23rd Cochrane Colloquium Jordan et al 2015)

$995 \times 705 \mathrm{~mm}(96 \times 96 \mathrm{DPI})$ 


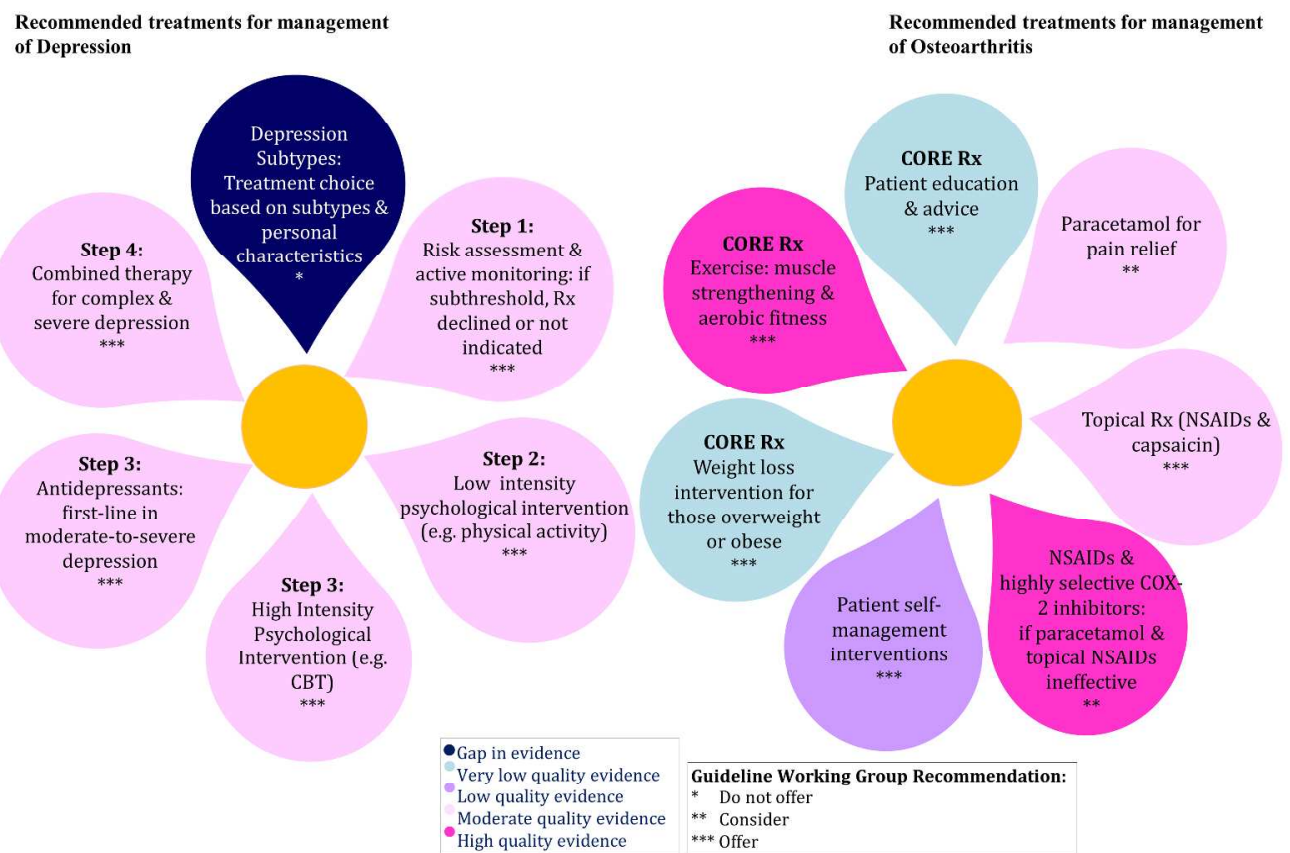

Figure 4: Evidence flowers illustrating Guideline recommendations for the management of Osteoarthritis and Depression

Figure 4: Evidence flowers illustrating Guideline recommendations for the management of Osteoarthritis and Depression

$995 \times 705 \mathrm{~mm}(96 \times 96 \mathrm{DPI})$ 